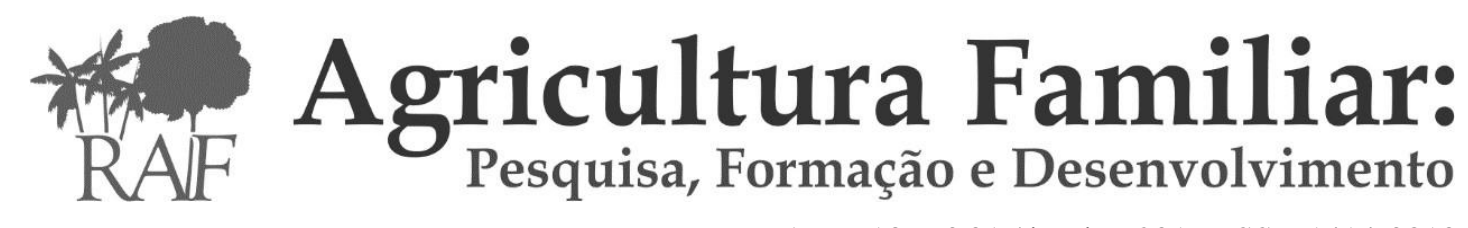

RAF. v.13, nº 01 / jan-jun 2019, ISSN 1414-0810

\title{
Resumos de Dissertações
}

\section{LÓGICAS FAMILIARES DE PRODUÇÃO, CONSUMO E AQUISIÇÃO DE RENDA FAMILIAR: INTERFERENCIA NA SEGURANÇA ALIMENTAR DA COMUNIDADE QUILOMBOLA NOSSA SENHORA DO LIVRAMENTO, MUNICÍPIO DE IGARAPÉ-AÇU, PARÁ}

\author{
YORGANA CAROLINA YAJURE PRADO
}

A segurança alimentar é um tema que vem sendo discutido por diversos países e instituições a nível nacional e internacional. A busca de avanços e soluções neste assunto continua sendo uma realidade inegável no mundo, sobretudo entre grupos tradicionais como os quilombolas que, devido a processos históricos, geográficos, socioeconômicos e/ou ambientais, apresentam vulnerabilidade ao risco alimentar. Esta pesquisa analisou as estratégias aplicadas pelas famílias da Comunidade Quilombola Nossa Senhora do Livramento para a obtenção de alimentos e sua interferência no estado da segurança alimentar das famílias estudadas. A amostra foi constituída por 25 famílias atendidas e não atendidas ao Programa de Fomento às Atividades Produtivas Rurais (MDSA-SEAD). Para a obtenção dos dados, optou-se pelo uso de entrevistas (estruturadas, semiestruturas e abertas); Diagnóstico Rápido Participativo (DRP); Linha do Tempo; Turnê Guiada; e Indicadores de Segurança Alimentar. A condição de segurança alimentar das famílias depende diretamente das políticas sociais do Governo Federal (Bolsa Família e aposentadorias) e da diversificação das estratégias (lógicas) utilizadas para aquisição de renda familiar mensal, que geralmente obedecem a trabalhos do tipo "bico" ou àquelas denominadas pelas famílias de "trabalhos fora da propriedade". A produção para o autoconsumo perdeu forças entre os grupos familiares da comunidade, incrementando o consumo de alimentos industrializados e o deslocamento das atividades próprias da cultura quilombola, interferindo nas atividades produtivas e na fragmentação das relações internas e externas da comunidade, que leva, indiscutivelmente, os grupos familiares a encontrarem-se dentro de uma situação desfavorável de segurança alimentar.

Palavras-chave: comunidades tradicionais, insegurança alimentar, agricultura familiar, Nordeste Paraense, Projeto Tipitamba.

Número de páginas: 140

\section{Banca Examinadora:}

Dr. Osvaldo Ryohei Kato (EMBRAPA)

Dr. ${ }^{a}$ Tatiana Deane de Abreu Sá (EMBRAPA)

Dr. William Santos de Assis (UFPA)

Dr. ${ }^{a}$ Celia de Azevedo (EMBRAPA)

\section{Local e Data de Defesa:}

EMBRAPA Amazônia Oriental, no dia 27 de agosto de 2018. 


\title{
IDEIAS, INTERESSES E INSTITUCIONALIZAÇÕES: PROPOSTAS DE FORTALECIMENTO DA AGROECOLOGIA NOS TERRITÓRIOS DA CIDADANIA NO ESTADO DO PARÁ
}

\author{
VALDIR DA CRUZ RODRIGUES
}

Este estudo teve como objetivo a identificação e análise de propostas de fortalecimento da agroecologia em Territórios da Cidadania. O universo do estudo se deu no âmbito dos territórios Baixo Tocantins, Nordeste Paraense e Sudeste Paraense. A abordagem de tratamento foi predominantemente qualitativa, tendo como referência de análise o enfoque dos 3I - Ideias, Interesses e Institucionalização. Para isso, se utilizou de entrevistas semiestruturadas, roteiro de pesquisa e observação participante. Analisou-se os processos que resultaram na concepção de ideias agroecológicas, suas lógicas de construção e a realidade de execução de propostas de investimento nos territórios institucionais comparando-as com as ações desenvolvidas nos territórios de ação coletiva dos atores locais. Isso permitiu tirar ensinamentos sobre as formas e as realidades de institucionalização da agroecologia nos três territórios, no sentido de sua forma de consolidação e perenização a partir dos jogos do atores. Com base na análise das informações e na realidade da agroecologia constatou-se uma diversidade de ideias acerca de quais percepções de agroecologia são evidenciadas pelos diferentes atores. A partir de um dispositivo de governança territorial - o CODETER - essas ideias se traduziram em propostas agroecológicas que foram inclusas em Planos Territoriais (PTDRS), cujas dimensões foram mobilizadas pelos Projetos Territoriais (PROINFs) em favor a agroecologia. Para isso, emergiram disputas de interesses e jogos de poder em torno dessas proposições e de outras dinâmicas locais que estruturam os modos de institucionalização. De fato, o estudo mostra esses territórios conseguiram institucionalizar referenciais e práticas agroecológicas em várias dimensões, com diferenças significativas entre os territórios de ação pública e os territórios de ação coletiva. Perspectivas sobre a ação pública ligada à agroecologia e sua relação com o enfoque territorial são destacadas.

Palavras-chave: Agroecologia. Território. Planejamento. Poder.

Número de páginas: 192

\section{Banca Examinadora:}

Dr. Marc Piraux (CIRAD/UFPA)

Dr. Carlos Douglas de S. Oliveira (UFRA)

Dr. William Santos de Assis (UFPA)

Dr. Carlos Valério Aguiar Gomes (UFPA)

\section{Local e Data de Defesa:}

INEAF, no dia 18 de março de 2019. 


\title{
PROCESSO ENSINO-APRENDIZAGEM NA ABORDAGEM ANALÍTICO COMPORTAMENTAL NO CONTEXTO DA AGRICULTURA FAMILIAR
}

\author{
RAFAEL PENICHE FERREIRA
}

As especificidades da gestão de um estabelecimento familiar requerem, por parte dos projetos de intervenção e da assistência técnica, compreensão e atuação que abarquem estas especificidades. Para além da análise global proposta na abordagem sistêmica, é igualmente importante acompanhar o nível de apropriação das informações técnicas ou não envolvidas no processo, a fim de melhorar os desempenhos finais. Neste estudo realizamos uma análise do processo de ensino-aprendizado, com base na teoria de Skinner, ocorrido durante a execução de um projeto de intervenção voltado para agricultores familiares produtores de leite em Paragominas. A pesquisa investigou a relevância da abordagem comportamental aplicado ao processo de ensinoaprendizagem, buscando compreender os benefícios destas para os profissionais que atuam no meio rural. Para tanto, foi utilizado uma abordagem quantiqualitativa e aplicações de questionários em campo baseados e adaptados para o método de Donald Kirkpatrick. Esse método é formado por quatro níveis, (primeiro nível - análise da satisfação (Reação); segundo - análise da aprendizagem (Aprendizagem); terceiro nível - análise comportamental (Comportamento) e o ultimo análise das mudanças (Resultado), diferentes avaliações. Os principais resultados apontaram que o conhecimento prévio consolidado influi no processo de ensino-aprendizagem, pois afeta o reconhecimento de informações, e consequentemente a capacidade de aprender, ou consolidar informações novas. De forma geral os agricultores apropriaram-se bem das informações tratadas, plantio, isolamento e tamanho da área, e apontaram fragilidades em duas etapas do processo: informações sobre divisão e uso das áreas de pastos. Segundo a literatura pesquisada, a dificuldade de compreensão pode ser decorrente da falta do conhecimento prévio.

Palavras-chave: Behaviorismo. Pastagens. Agricultores familiares. Aprendizagem. Projeto de intervenção.

Número de páginas: 100

\section{Banca Examinadora:}

Dr. ${ }^{a}$ Laura Angélica Ferreira Darnet (UFPA)

Dr. ${ }^{a}$ Olivia Misae Kato (UFPA)

Dr. René Poccard-Chapuis (CIRAD-EMBRAPA)

\section{Local e Data de Defesa:}

INEAF, no dia 03 de abril de 2019. 


\title{
ENTRE O CAMPO E A CIDADE: O JOVEM RIBEIRINHO E SUAS RELAÇÕES COM O PROCESSO DE MIGRAÇÃO NA REGIÃO DAS ILHAS DE ABAETETUBA/PA
}

\author{
EDILCINA MONTEIRO FERREIRA
}

Neste estudo é apresentado os anseios de jovens ribeirinhos de algumas comunidades das ilhas do município de Abaetetuba/PA. Anseios por um futuro com estabilidade financeira para si e seus familiares. Anseios por uma vida na cidade onde poderão dar continuidade aos estudos e ter uma boa formação, almenjando emprego com carteira assinada. É apresentado ainda a realidade de jovens que desejam permanecer no campo dando sucessão ao trabalho praticado pela família durante décadas, que desejam viver na calmaria do meio rural e livre da correria de quem vive na cidade. São apresentados os principais motivos que levam os jovens ribeirinhos a desejarem sair do campo em direção a cidade, e a realidade da migração do gênero feminino e a masculinização do campo. Foram realizadas 45 entrevistas semiestruturadas, organizadas através de questionários, aplicados com jovens das comunidades visitadas, os jovens entrevistados foram localizados nas comunidade (jovens que ainda residem nas ilhas) e também na cidade (jovens que são das comunidades das ilhas, mas residem atualmente na cidade de Abaetetuba/PA), a pesquisa apresenta uma abordagem qualitativa. Além destas, também foram realizadas 9 entrevistas direcionadas as famílias de jovens em três comunidades (Sagrado Coração de Jesus, Santa Maria e Bom Jesus), nesse momento utilizou-se o método de pesquisa participante, que consiste numa investigação efetivada a partir da inserção e interação do pesquisador(a) no grupo, comunidade ou instituição investigada. Na área de várzea os jovens auxiliam nas atividades domésticas e nas plantações, a principal fonte de renda das famílias está no período da safra do açaí, quando os ribeirinhos colhem os frutos e vendem na cidade ou para atravessadores. Nesse período que os jovens ganham dinheiro, colhendo os frutos e ajudando os pais na comercialização, mas é também nesse período que surgem as evasões de jovens da escola, uma situação muito frisada por alguns entrevistados. Este trabalho buscou mostrar a diferença de gêneros frente a migração e os motivos que levam homens e mulheres a migrarem para a cidade.

Palavras-chave: Juventude. Ribeirinhos. Migração. Mobilidade.

Número de páginas: 92

\section{Banca Examinadora:}

Dr. ${ }^{a}$ Angela May Steward (UFPA)

Dr. Carlos Augusto Cordeiro Costa (UFRA)

Dr. ${ }^{a}$ Ruth Helena Cristo Almeida (UFRA)

\section{Local e Data de Defesa:}

INEAF, no dia 29 de abril de 2019. 


\title{
TRAJETÓRIA DE INSTITUCIONALIZAÇÃO DA AGROECOLOGIA NO NORDESTE PARAENSE: AS EXPERIÊNCIAS DE IRITUIA E SANTA LUZIA DO PARÁ
}

\author{
ELLY CRYSTIAN DE OLIVEIRA PINTO
}

A presente pesquisa teve como objetivo explicar o nível atual de Consolidação da Agroecologia em duas experiências no Nordeste Paraense. Para tal, foram analisados e comparados processos de Institucionalização da Agroecologia nos municípios de Irituia e Santa Luzia do Pará. A construção da pesquisa se deu a partir do estudo da trajetória de institucionalização, para descrever o grau de Institucionalização da Agroecologia, comparar trajetórias e identificar fraquezas e potencialidades em ambos os municípios. Para isso, fizemos a análise bibliográfica, análise de documentos, entrevistas não diretivas e oficina de intercâmbio, com a finalidade de verificar a percepção e o discurso dos atores acerca do avanço da transição agroecológica e sua consolidação. Chegou-se à conclusão que a Agroecologia encontra-se consolidada em ambos os municípios, devido ao processo de Institucionalização nas suas diferentes dimensões, devido à articulação de atores como agricultores familiares e suas instituições, a introdução de mulheres e jovens na agroecologia, assim como, o acesso a novos mercados e políticas públicas. Contudo, verificamos que é necessário avançar no diálogo com a gestão local, renovação dos atores e aproximação com movimentos como STTRS, intercâmbios e sistematização das experiências, ação coletiva (fundamental), consolidação das feiras.

Palavras-chave: Juventude. Ribeirinhos. Migração. Mobilidade.

Número de páginas: 92

\section{Banca Examinadora:}

Dr. Marc Piraux (CIRAD/UFPA)

Dr. ${ }^{a}$ Tatiane Deane de Abreu Sá (EMBRAPA)

Dr. William Santos de Assis (UFPA)

Dr. Carlos Valério Aguiar Gomes (UFPA)

\section{Local e Data de Defesa:}

INEAF, no dia 13 de maio de 2019. 


\title{
A CONTRIBUIÇÃO DOS NÚCLEOS DE ESTUDOS EM AGROECOLOGIA (NEAS) PARA O ENSINO, PESQUISA E EXTENSÃO EM INSTITUIÇÕES NO NORDESTE PARAENSE
}

\author{
MARIA JOSÉ BRITO PINHEIRO
}

Este trabalho tem como objetivo refletir sobre a contribuição dos núcleos de estudos em agroecologia no estado do Pará para os processos de ensino-pesquisa-extensão nas instituições de ensino e pesquisa no nordeste paraense. Fundamentamos o trabalho em princípios da agroecologia, do desenvolvimento sustentável, da construção do conhecimento agroecológico e da indissociabilidade entre ensino-pesquisa-extensão. Realizamos a pesquisa em quatro núcleos de estudos em agroecologia: NEA IFPA-Castanhal, NEA Ajuri-UFPA Belém, NEA UFRACapitão Poço e o NEA Puxirum Agroecológico-Embrapa Amazônia Oriental. Utilizamos uma abordagem qualitativa apoiada pela pesquisa bibliográfica, pesquisa documental, pesquisa de campo, entrevistas semiestruturada e a utilização do questionário. Como resultado nós interferimos que os núcleos de estudos em agroecologia vêm desenvolvendo ações relevantes em torno do processo de construção do conhecimento agroecológico no nordeste paraense, refletindo também em outros territórios do estado. Os núcleos de estudos em agroecologia apresentam esforços significativos para levar a cabo os processos indissociáveis entre o ensino, a pesquisa e a extensão. Com características institucionais distintas, os núcleos de estudos em agroecologia se constituem em espaços de debate sobre os princípios da agroecologia e funcionam como ferramentas importantes para a articulação entre o conhecimento científico acadêmico e o saber das comunidades e povos do campo.

Palavras-chave: Agroecologia; Saberes locais; Construção de conhecimentos; Comunidades rurais; Nordeste do Pará.

Número de páginas: 134

\section{Banca Examinadora:}

Dr. William Santos de Assis (UFPA)

Dr. Fabiano de Oliveira Bringel (UEPA)

Dr. Marc Piraux (CIRAD-UFPA)

Dr. Carlos Valério Aguiar Gomes (UFPA)

\section{Local e Data de Defesa:}

INEAF, no dia 07 de junho de 2019. 


\title{
NARRATIVAS COSMOLÓGICAS REGISTRADAS NA MATA DO BACURIZAL NO QUILOMBO BAIRRO ALTO, SALVATERRA (PA)
}

\author{
MAYARA GONÇALVES LIMA
}

Esta pesquisa de mestrado se debruçou em analisar as relações produtivas do trabalho com o bacuri, e a relação entre a sociedade e a natureza da Comunidade Quilombola Bairro Alto, pertencente à cidade de Salvaterra, que faz parte do arquipélago fluviomarinho do Marajó (PA). A ilha passou por diversas ocupações ao longo dos séculos inicialmente as etnias indígenas, e posteriormente com a colonização europeia houve a diáspora africana, no qual os africanos foram trazidos para trabalhar em fazendas de gado. As comunidades quilombolas marajoaras se constroem a partir das relações étnicas no arquipélago, assim como a organização politica nacional de outros quilombos, atravessaram a comunidade estudada, e ao longo dos anos reivindicam as leis principalmente as ligadas à titulação definitiva das suas terras, devido os conflitos territoriais com fazendeiros, a EMBRAPA, entre outros. As áreas em disputas são importantes para a reprodução econômica dos quilombos, os trabalhos com coleta dos recursos naturais, nas roças, matas, rios, mares, campos, entre outros ecossistemas garantem a manutenção das unidades familiares, a pesquisa se debruçou em analisar justamente o trabalho com a coleta do bacuri, os saberes tradicionais usados nas matas dos onde estão as árvores do bacuri revelam as cosmovisões das comunidades. A pesquisa antropológica efetuada metodológica se baseou na etnografia aliada à observação participante nas casas das famílias, entrevistas, foetnografia, e nas vivências nas matas, para compreender a rotina das famílias e como se organizavam para o trabalho nos bacurizais, as entrevistas e fotos complementam os dados coletados.

Palavras-chave: Marajó. Quilombo. Economia doméstica. Cosmologia.

Número de páginas: 107

\section{Banca Examinadora:}

Dr. ${ }^{a}$ Angela May Steward (UFPA)

Dr. Flávio Bezerra Barros (UFPA)

Dr. Maria das Graças da Silva (UEPA)

Dr. Carlos Valério Aguiar Gomes (UFPA)

\section{Local e Data de Defesa:}

INEAF, no dia 11 de junho de 2019. 


\title{
TRAJETÓRIA DA REFORMA AGRÁRIA AMBIENTAL NA AMAZÔNIA: AVALIAÇÃO DE RESULTADOS EM PROJETOS DE DESENVOLVIMENTO SUSTENTÁVEL EM ANAPU, SUDOESTE DO PARÁ
}

\author{
MARA CRISTINA DE LIMA COSTA
}

O trabalho apresenta resultados da pesquisa que teve como objetivo analisar a trajetória de implementação da modalidade de assentamento Projeto de Desenvolvimento Sustentável-PDS no Sudoeste do Pará, a fim de constatar a efetiva ocorrência de iniciativas ambientalmente diferenciadas. O recorte da pesquisa compreende o município de Anapu, pertencente a região da Transamazônica-PA, que devido ao modelo de colonização imposto pelo governo ocasionou diversos conflitos e tensões, principalmente em função da disputa pela posse da terra e do acesso a outros recursos naturais. Diante desses conflitos surge na região, por meio da luta dos movimentos sociais, a modalidade de assentamento PDS, como uma nova proposta diante dos problemas decorrentes dos assentamentos convencionais. Para a pesquisa foram utilizados métodos qualitativos e quantitativos. A coleta de dados foi realizada por meio de entrevistas semiestruturadas, diagnóstico socioeconômico e realização de reuniões com grupos focais, o que possibilitou uma analise comparativa entre a modalidade de assentamento convencional e os PDS. Entre os principais resultados destacou-se que apesar da maior consciência ambiental entre os assentados dos PDS, as iniciativas ambientalmente sustentáveis ainda não são uma realidade nesses assentamentos. Os resultados do estudo concluem que a implementação de assentamentos ambientalmente diferenciados, apesar de ser proposta inovadora, não se concretiza de modo a alcançar o desenvolvimento proposto pela modalidade, sendo necessárias melhorias e maior investimento nas ações.

Palavras-chave: Reforma agrária, Projetos de assentamento, Desenvolvimento sustentável.

Número de páginas: 210

\section{Banca Examinadora:}

Dr. Roberto Porro (EMBRAPA)

Dr. Alfredo Kingo Oyama Homma (EMBRAPA)

Dr. Maurício Torres (UFPA)

Dr. ${ }^{a}$ Noemi Sakiara Miyasaka Porro (UFPA)

\section{Local e Data de Defesa:}

INEAF, no dia 21 de junho de 2019. 


\section{“O QUE MUDOU?”: ESTUDO DAS TRAJETÓRIAS DOS SISTEMAS AGROFLORESTAIS NO MUNICÍPIO DE IRITUIA-PA}

\section{LAYSE DE NAZARÉ GONZAGA BRAGA GALVÃO}

Esta dissertação trata das trajetórias dos sistemas de produção dos agricultores familiares que trabalham com SAFs no município de Irituia, identificando as condições internas e externas que os levam a introduzir/modificar o SAF. A pergunta que mobiliza a pesquisa é: Como evoluiu, no tempo, o papel dos SAFs nas lógicas familiares de produção, no município de Irituia, Nordeste Paraense? O estudo foi realizado no município de Irituia no ano de 2018. Para compreender as mudanças ocorrentes no sistema de produção foi feita uma análise multi escalar, do contexto agrário e dos estabelecimentos agrícolas, com base no enfoque sistêmico. Para tanto, utiliza-se como método e ferramentas a entrevista histórica, análise documental, análise de paisagem, aplicação de questionários e a análise retrospectiva. Os resultados são apresentados em dois artigos: I - o primeiro artigo identifica os eventos históricos e as mudanças nos fatores externos ao sistema de produção que condicionaram suas transformações para a introdução/ modificação dos SAFs. Verificou-se que os SAFs em Irituia foram consolidados a partir, principalmente, de incentivos endógenos provenientes de ações locais que estimularam a sua expansão/modificação dentro do sistema de produção. Políticas públicas que ocorreram na história, como o PROAMBIENTE, o PAA e o PNAE, a organização social e a estruturação da infraestrutura do município têm estimulado e valorizado a diversificação do SAF e do próprio sistema de produção. II - o segundo artigo analisa as transformações ocorridas nos sistemas de produção ao longo do tempo e as adaptações necessárias para introdução/ modificação dos SAFs. Constatou-se que os agricultores adequam seus sistemas de acordo com as oportunidades, interesses próprios e da família em um dado momento da história. Percebe-se que as trajetórias dos sistemas de produção passam por um período em que a roça entra em crise e, por este e por outros motivos, o SAF vai ganhando espaço nos estabelecimentos agrícolas familiares e no município. De um modo geral, as trajetórias dos sistemas de produção compreendem fases de transformação, de diversificação e agregação de valor, tanto no SAF quanto no sistema de produção como um todo. Há sinalização de recuperação florestal nos estabelecimentos pela prática do SAF e conservação de capoeiras ocasionada pela apicultura. Conclui-se que a expansão do SAF em Irituia e a diversificação do sistema de produção foram possíveis graças ao desenvolvimento estrutural, os interesses e apoio do poder local e o empenho dos agricultores. A inserção dos SAFs nos sistemas de produção proferiu mudanças para além da estrutura do sistema de produção, ocasionando a criação da cooperativa, a mudança da relação dos agricultores com a natureza e a construção de uma nova identidade.

Agricultura Familiar: Pesquisa, Formação e Desenvolvimento • Belém • v.13 , nº1 • p. 120-129 • jan-jun 2019 
Palavras-chave: sistemas agroflorestais. agricultura familiar. sistemas de produção. contexto agrário. trajetórias

Número de páginas: 114

\section{Banca Examinadora:}

Dr. Lívia de Freitas Navegantes Alves (UFPA)

Dr. Luís Mauro Santos Silva (UFPA)

Dr. ${ }^{a}$ Luciane Cristina Costa Soares (UFRA)

Dr. ${ }^{a}$ Angela May Steward (UFPA)

\section{Local e Data de Defesa:}

INEAF, no dia 18 de junho de 2019. 